\title{
Quantum Reflection from an Oscillating Surface
}

\author{
Benedikt Herwerth $^{1}$, Maarten DeKieviet ${ }^{2}$, Javier Madroñero ${ }^{3}$, \\ Sandro Wimberger ${ }^{1}$ \\ 1 Institut für Theoretische Physik, Universität Heidelberg, Philosophenweg 19, \\ D-69120 Heidelberg, Germany \\ 2 Physikalisches Institut, Universität Heidelberg, Im Neuenheimer Feld 226, D-69120 \\ Heidelberg, Germany \\ 3 Departamento di Física, Universidad del Valle, Cali, Colombia
}

\begin{abstract}
We describe an experimentally realistic situation of the quantum reflection of helium atoms from an oscillating surface. The temporal modulation of the potential induces clear sidebands in the reflection probability as a function of momentum. Theses sidebands could be exploited to slow down atoms and molecules in the experiment.
\end{abstract}

\section{Introduction}

Periodically driven quantum system are natural working horses in various fields of physics. For low-dimensional systems, they provide paradigms of classical and quantum chaos [1-4]. From the mathematical point of view, the temporal periodicity allows for the application of the Floquet theorem 2 to arrive at effectively time-independent quantum problems which is accessible to analytical treatment, see e.g. [5], or may be simply diagonalized numerically, see e.g. [6,7]. Practically, temporal periodicity is naturally given by oscillators, e.g. electromagnetic or mechanical waves, providing the external drive.

In this paper we study the reflection of helium atoms from an attractive potential. Since the reflection can then only be caused by quantal effects, this problem is generally dubbed quantum reflection. Quantum reflections have been experimentally investigated over the last decade by various groups, see e.g. [8-12], and they are also relevant in the context of hybrid quantum systems [13] involving ultracold atoms bouncing from nanostructures [14-18]. As an extension of these experiments with static barriers, we here propose the experimental implementation of a situation where the atoms interact with an oscillating barrier [19]. The modulation is assumed to be sinusoidally periodic, which may be realized using a vibrating nanowire [17] or simply a commercially available vibrating membrane in the laboratory [20].

We use a refined numerical method to propagate initial atomic wave packets toward the membrane and investigate their reflection probability. The latter shows characteristic sidebands induced by the periodic modulation. These sidebands can 
be clearly resolved for typical experimental parameters. The controlled addressing of the sidebands allows the experimentalist to engineer the reflection probability and the momentum of the atoms. Our numerical integration scheme restricts to one spatial dimension, but it is very efficient. This provides a proof-of-principle for the possibility to compute precise reflectivities also for higher-dimensional setups.

\section{Theoretical background and numerical method}

A possible way to describe the long-range atom-surface interaction is given by the phenomenological Casimir-van der Waals potential [8,9,21]

$$
V(x)=-\frac{C_{4}}{x^{3}(x+l)},
$$

where $x$ is the distance from the atom to the surface and $l$ is the reduced wavelength of the atomic transition. For $x \ll l$, the potential is proportional to $-1 / x^{3}$, whereas for $x \gg l$ retardation effects are taken into account by the $-1 / x^{4}$-behavior. The values used throughout this paper correspond to the transition wavelength of helium $l=93 \AA$ and the interaction between helium and a silicon surface $C_{4}=23.25 \mathrm{eV} \AA^{4}$ [9, 10].

An atom, which is not reflected in the long-range regime and, therefore, approaches the surface up to a distance of some atomic units, can be subject to inelastic reflections or to sticking processes. Since we only want to consider the quantum reflection from within the long-range regime, a regularization of the potential is introduced, which allows particles coming close to the surface to move further to $x \rightarrow-\infty$. Therefore, the potential is cut at a point $x_{0} \gtrsim 0$ and suitably continued for $x \leq x_{0}$. The reflectivity is then calculated for different values of $x_{0}$, which allowed to determine the reflectivity for the quantum reflection by extrapolation to $x_{0} \rightarrow 0$. Practically, a parabolic curve was used to continue the potential for $0 \leq x \leq x_{0}$ and a constant potential for $x<0$. All parameters of this continuation are chosen such that the potential and its derivative are continuous in $x=x_{0}$ and in $x=0$ :

$V_{\text {cont }}(x)=\left\{\begin{array}{lr}V(x), & \text { if } x_{0}<x \\ V\left(x_{0}\right)+V^{\prime}\left(x_{0}\right)\left(x-x_{0}\right)+\frac{V^{\prime}\left(x_{0}\right)}{2 x_{0}}\left(x-x_{0}\right)^{2}, & \text { if } 0 \leq x \leq x_{0} \\ V\left(x_{0}\right)-\frac{1}{2} V^{\prime}\left(x_{0}\right) x_{0}, & \text { if } x<0\end{array}\right.$.

$V$ denotes the Casimir-van der Waals potential of Eq. (11) and $V^{\prime}$ its derivative. To describe the harmonically oscillating surface, the continued potential $V_{\text {cont }}$ is shifted harmonically along the $x$-axis. We thus use the following time-dependent version of the potential in the Kramers-Henneberger frame of reference [22] for our computations:

$$
W(x, t)=V_{\text {cont }}(x-d \sin (\omega t)) .
$$

Herein $d$ denotes the amplitude of the oscillation and $\omega$ its frequency.

For both the oscillating and the stationary potential $V_{\text {cont }}$, the one-dimensional, time-dependent Schrödinger equation is integrated numerically, using a Gaussian wave packet for the initial state. The evolution of the initial state is done by a norm 
preserving Crank-Nicolson scheme [23,24]. Comparing different order approximations of the Hamiltonian with respect to convergence and efficiency, a three-point finite-difference approximation of the Hamiltonian is finally used in our calculations. Especially when considering a time-dependent system, the three point approximation turned out to be most effective, because the matrix inversion needed for the time-evolution can be carried out by Gaussian elimination [23,24].

When integrating the time-dependent Schrödinger equation for a wave packet, artificial reflections arise when the wave packet hits the boundary of the numerical box. Especially for small values of the connection point $x_{0}$, the transmitted part of the wave function is subject to such reflections. Therefore, absorbing boundaries were introduced by multiplying the wave function $\psi(x)$ by a damping function $f(x)$ after each time step $\Delta t: \psi(x) \rightarrow f(x) \psi(x)$. This damping function is chosen such that it is unity (within numerical accuracy) for $x>0$ and that it falls off smoothly to positive values smaller than unity for $x<0$. Practically, the damping function

$$
f(x)=\frac{1}{\exp \left(-\frac{x-a}{\sigma}\right)+1},
$$

is used, where $a$ and $\sigma$ are the two parameters that determine the position and the sharpness of the damping function. If $x_{b}$ is the lower boundary of the numerical box, where the wave function is to be suppressed, $a$ and $\sigma$ were chosen such that the following conditions are fulfilled to minimize artificial reflections, see also Sec. 3; $f\left(x_{b}\right)=10^{-8}$ and $|f(x)-1|<10^{-16}$ for $x>0$.

In the case of the time-independent potential, our approach can be benchmarked with a more straightforward calculation. For this, the reflectivity is calculated by a numerical integration of the time-independent Schrödinger equation for a particle with energy $E=\hbar^{2} k^{2} / 2 m$. The boundary condition for the stationary wave function $\phi$ is chosen such that for $x \rightarrow-\infty$ a left-going, transmitted particle is represented by $\phi$. Therefore, a left-going plane wave is used to define an initial condition $\left(\phi\left(x_{i}\right), \phi^{\prime}\left(x_{i}\right)\right)$ at $x_{i} \leq 0$, where the potential (in the continued form) is constant and plane waves are exact solutions to the Schrödinger equation. By numerical integration the wave function and its derivative were obtained at a point $x_{f} \gg 0$, where the potential approximately vanishes. The reflectivity is then calculated by matching the wave function at $x_{f}$ to the sum of an incoming and an outgoing plane wave:

$$
\begin{aligned}
& \phi\left(x_{f}\right)=A e^{i k x_{f}}+B e^{-i k x_{f}} \\
& \phi^{\prime}\left(x_{f}\right)=i k A e^{i k x_{f}}-i k B e^{-i k x_{f}} .
\end{aligned}
$$

We solve Eq. (5) for the constants $A$ and $B$ and compute the reflectivity as $R=|A / B|^{2}$. The adaptive-stepsize Runge-Kutta integrator odeint of the SciPy-software package [25] is used to calculate $\left(\phi\left(x_{f}\right), \phi^{\prime}\left(x_{f}\right)\right)$ numerically. 


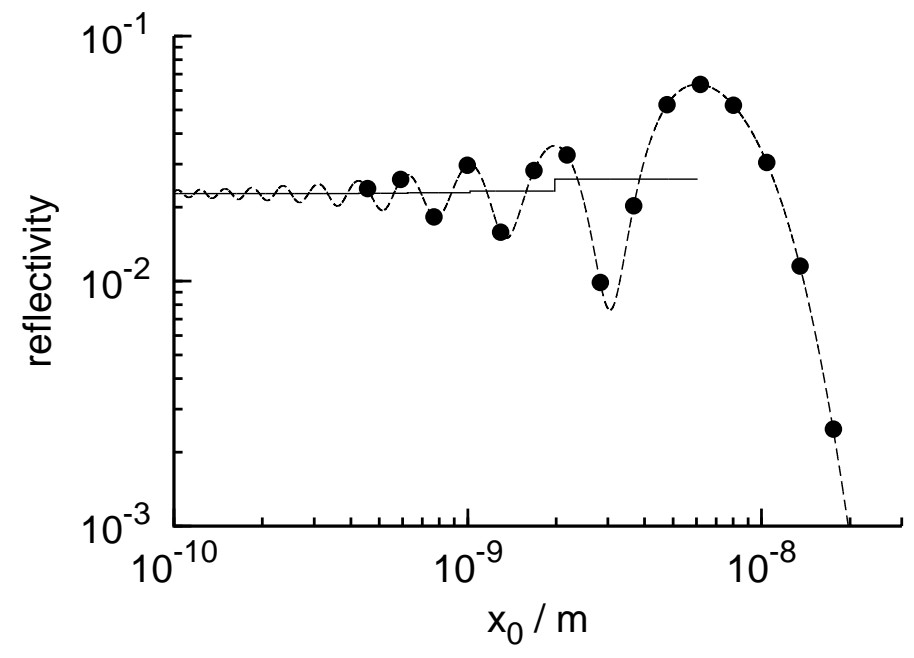

Figure 1. Reflectivity for the static potential as a function of $x_{0}$. Results obtained by integrating the time-dependent Schrödinger equation (filled circles) and by solving the stationary Schrödinger equation (dashed line) are shown. The solid line shows the averages calculated between subsequent maxima by double-geometric averaging.

\section{Results}

Static Potential. Inspired by the experimental setup reported in [9, 12, 26], we consider ${ }^{3} \mathrm{He}$ atoms with mass $m=3.01603 \mathrm{u}$. The reflectivity is first calculated for the static potential of Eq. (2) by integration of the time-dependent and the time-independent Schrödinger equation. For the time-dependent integration, the initial state is a Gaussian wave packet with a mean velocity of $v=-2 \mathrm{~m} / \mathrm{s}$ and a relative standard deviation of $\Delta v / v=3 \%$. The initial wave packet is placed at $x=4.5 \mu \mathrm{m}$. The wave packet is propagated until the reflected part of the momentum distribution becomes stationary. This occurs at times $t<3.4 \mu \mathrm{s}$. At $t=3.4 \mu \mathrm{s}$, a Fourier transform of $\psi(x)$ is carried out (efficiently implemented using Fast Fourier Transforms [23]) to obtain the momentum distribution $\psi(k)$. The reflectivity is calculated by integrating the probability distribution of the reflected particle $|\psi(k)|^{2}$ in momentum space.

Fig. 1 1 shows the calculated reflectivity as a function of the connection point $x_{0}$. We observe an oscillating behavior around an asymptotic stationary value, which is reached only in the limit $x_{0} \rightarrow 0$. The oscillations can be interpreted as a consequence of the "artificial" connection at $x_{0}$. Since the amplitude of the oscillations decreases as $x_{0}$ reaches smaller values, the influence of the connection becomes less and less important for smaller values of $x_{0}$. The asymptotic reflectivity is best extrapolated by taking averages between subsequent maxima as shown in Fig. 1 by the solid line. For 60 values of $x_{0}$ between $4 \cdot 10^{-10} \mathrm{~m}$ and $2 \cdot 10^{-8} \mathrm{~m}$, the reflectivity is calculated in this way using the time-dependent and the time-independent method. The values obtained by integration of the time-dependent Schrödinger equation are consistent with those obtained by the time-independent integration within a relative deviation of the order of one per cent. 


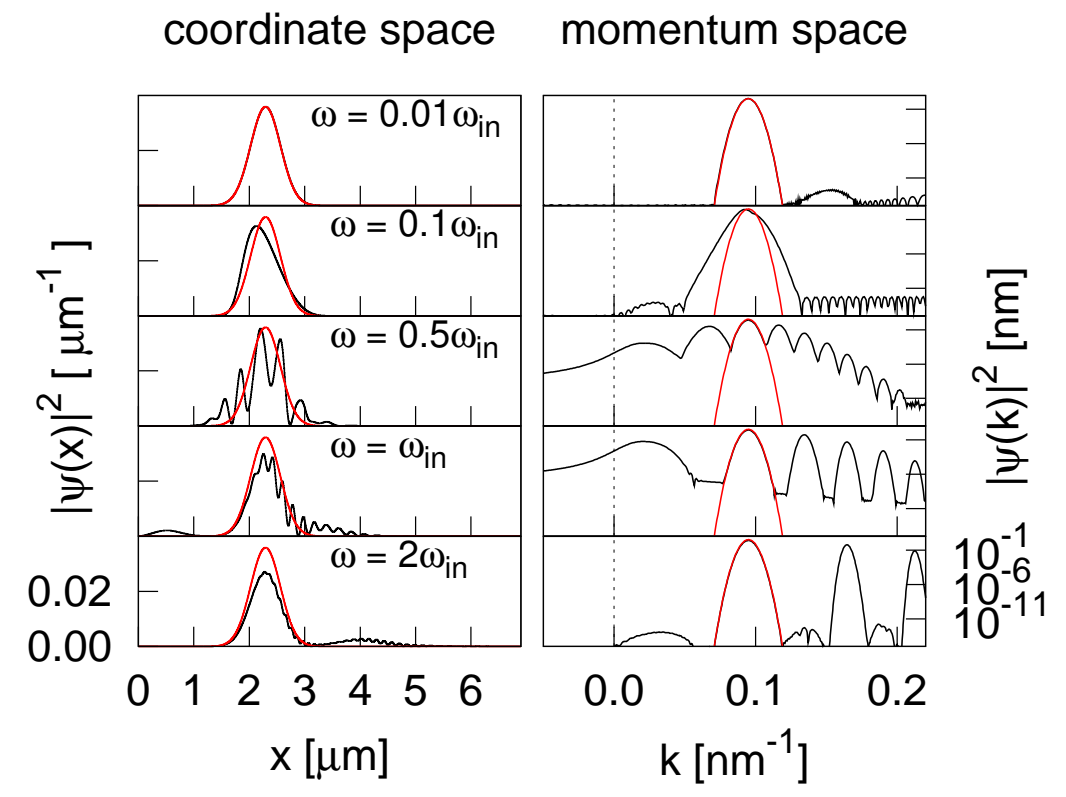

Figure 2. Snapshots of the outgoing wavepackets at $t=3.4 \mu \mathrm{s}$. Coordinate (in linear scale) and momentum distribution (in semilogarithmic scale) for a particle reflected on the oscillating surface (amplitude $d=4 \mathrm{~nm}$ ) for different frequencies $\omega$ and fixed $x_{0}=4 \times 10^{-10}$ (black curves). Red curves: corresponding distributions for the static potential.

Oscillating Surface. We now consider the dynamic potential of Eq. (3) describing the oscillating surface. A realistic value of $d=4 \mathrm{~nm}[20$ is chosen for the amplitude of the oscillation. If $\omega_{\text {in }}=E / \hbar$ is the energy of the incoming particle divided by $\hbar$, the oscillation of the surface is expected to influence the dynamics of the particle if the frequency $\omega$ is of the order of $\omega_{\text {in }}$. For the connection point $x_{0}=4 \times 10^{-10} \mathrm{~m}$ and a particle with initial velocity $v=2 \mathrm{~m} / \mathrm{s}$ and $\Delta v / v=3 \%$, the probability distribution of the reflected particle is calculated in momentum and in coordinate space for different values of $\omega_{\text {in }}$ as shown in Fig. 2 ,

For $\omega=0.01 \omega_{\text {in }}$, no effects are visible, whereas for $\omega=0.1 \omega_{\text {in }}$, distortions appear in the distributions. When $\omega$ reaches $0.5 \omega_{\text {in }}$, sidebands to the central peak in the momentum distribution become clearly visible. If the oscillation frequency is increased further beyond $\omega_{\text {in }}$, only the right sideband peaks remain. This occurs because the left side peak then corresponds to a negative momentum, i.e. to a transmitted particle, which is absorbed by the boundary. On the right hand side in Fig. 2, the right peaks moves further away from the central one with increasing frequency. This shows that the energy transfer becomes larger. In the coordinate distributions, significant oscillations imprinted by the moving surface are visible for $\omega \geq 0.5 \omega_{\text {in }}$.

The energy transfer due to the oscillation of the surface is expected to be quantized by integer multiples of $\hbar \omega$ :

$$
E_{n}=\hbar \omega_{\text {in }}+n \hbar \omega \text {. }
$$




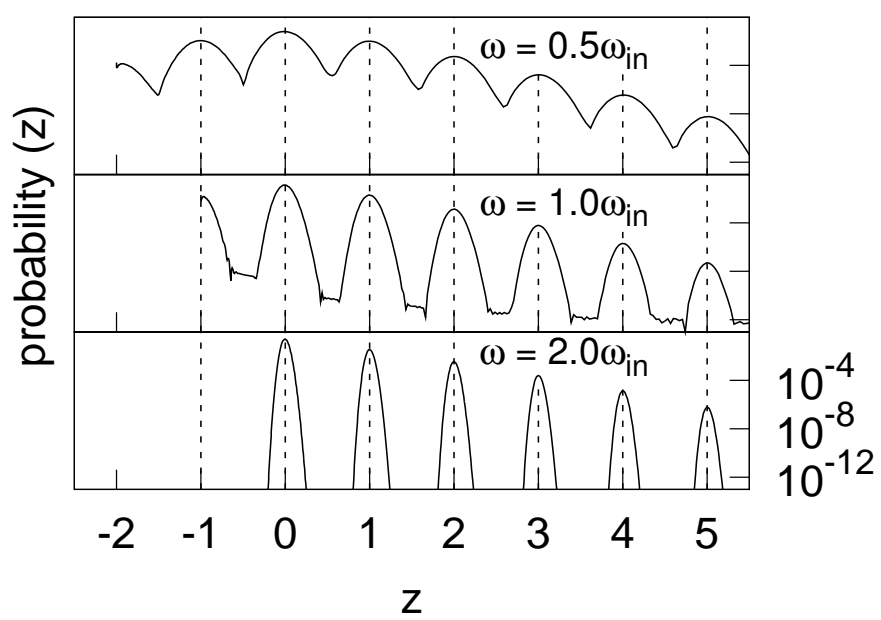

Figure 3. Rescaled and shifted energy distribution $\rho(z)$ of the reflected particle for the same parameters as in the previous figure. The distribution is computed from the momentum distribution for positive momenta $k$ only (see Fig. 2). For each value of $\omega$, the peaks occur at integer values of $z=n \in \mathbb{N}$, c.f. Eq. (7).

Because of the nonlinear dispersion relation, it is convenient to consider the distribution $\rho^{\prime}(k) \mathrm{d} k \equiv|\psi(k)|^{2} \mathrm{~d} k$ as a function of the new variable, c.f. also [19],

$$
\begin{aligned}
& z=\frac{\frac{\hbar^{2} k^{2}}{2 m}-\hbar \omega_{\text {in }}}{\hbar \omega}, \\
& \rho^{\prime}(k) \mathrm{d} k=\rho^{\prime}(k) \frac{m \omega}{\hbar k} \mathrm{~d} z=\rho(z) \mathrm{d} z .
\end{aligned}
$$

The peaks in this distribution $\rho(z)$ should now be at integer values of $z$. As shown in Fig. 3, this expectation is fulfilled, which confirms that the observed side peaks arise from the energy transfer between the particle and the oscillating surface.

To estimate the reflectivities $R_{n}$ for the different peaks of order $n$ in the momentum distribution in the presence of the oscillation, calculations for different values of $x_{0}$ were carried out for $\omega=0.5 \omega_{\text {in }}$. As in the case of the non-oscillating surface (Fig. 1), the reflectivity for the central peak, $R_{0}$, and for the two first order side peaks, $R_{-1}$ and $R_{1}$, shows an oscillating behavior as function of $x_{0}$. Their final value is obtained by taking the average of the reflectivity as a function of $x_{0}$ between two subsequent maxima, c.f. the solid line in Fig. 1. The calculated reflectivity for $R_{0}$, which corresponds to an elastic reflection in the laboratory frame, and for $R_{1}$ and $R_{-1}$, corresponding to an energy transfer of $\pm \hbar \omega$, are shown in Fig. 4 for three incident velocities.

Our calculations show that the effects caused by the oscillations of the surface are present over a large range of incident velocities. For a small incident velocity $(v=0.2 \mathrm{~m} / \mathrm{s})$, the reflectivity for the central peak dominates. For larger incident velocities, the side peaks gain importance. At $v=6 \mathrm{~m} / \mathrm{s}$, the sidebands even dominate the central peak. For $v=2 \mathrm{~m} / \mathrm{s}$ the reflectivity for the side peaks contributes approximately $13 \%$ to the total reflectivity. For $v=6 \mathrm{~m} / \mathrm{s}$, it is more likely for the 


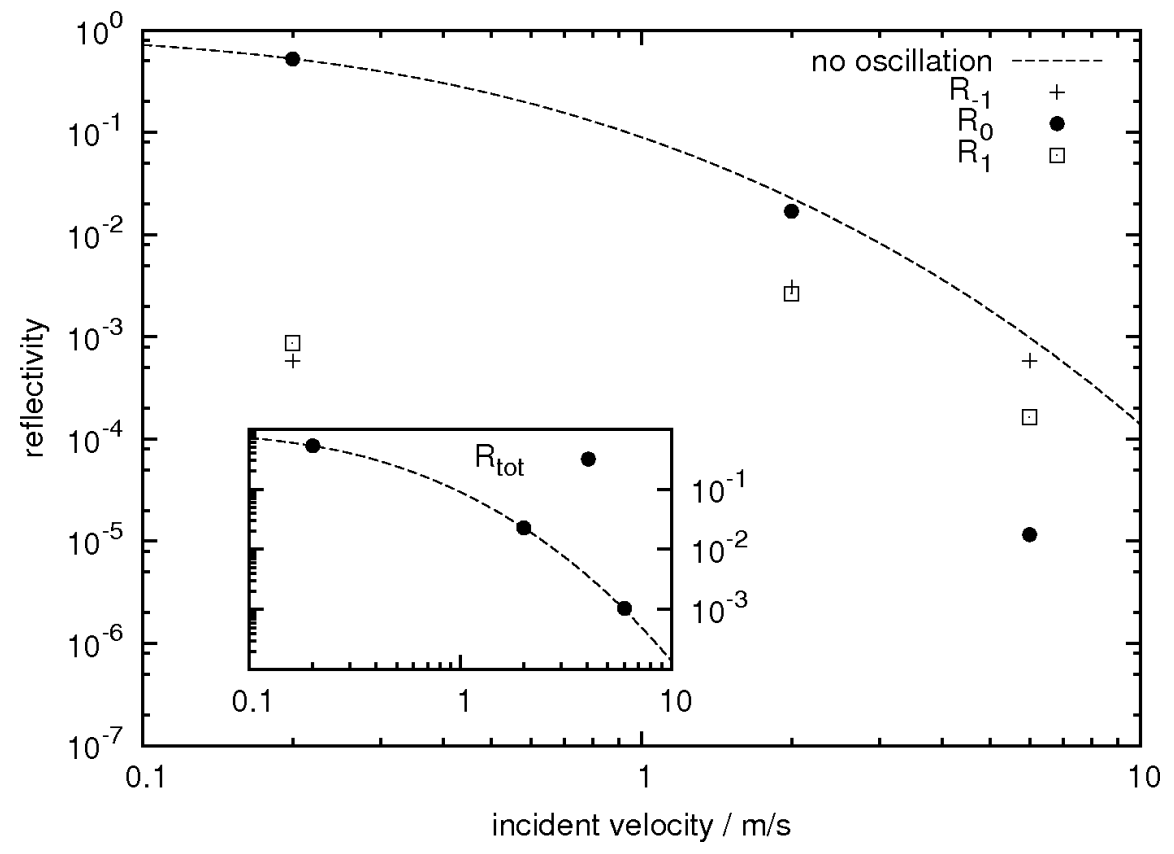

Figure 4. Reflectivity in the presence of the oscillation for $\omega=0.5 \omega_{\text {in }}$. The reflectivity for the central peak $R_{0}$ and the two side peaks $R_{-1}$ and $R_{1}$ is shown, together with the reflectivity for the non-oscillating surface for comparison (dashed line). To calculate the $R_{-1}, R_{0}$ and $R_{1}, 60$ calculations between two subsequent maxima in the reflectivity as a function of $x_{0}$ were carried out in the range $3.7 \cdot 10^{-10} \mathrm{~m}<\mathrm{x}_{0}<7.3 \cdot 10^{-10} \mathrm{~m}$ for each velocity. Inset: Reflectivity for the non-oscillating surface (dashed line) and total reflectivity $R_{\text {tot }}$ for the oscillating surface (filled circles).

particle to lose than to gain energy $\left(R_{-1}>R_{1}\right)$. This is interesting for practical purposes since it may be used to preferentially slow down atoms or molecules.

As a consistency check, we finally compute the total reflectivity for the three velocities considered in Fig. 4. This is done by integrating the momentum distribution of the reflected particle around the central and the contributing sidebands. The calculated reflectivity is compared to the one for the non-oscillating surface in Fig. 4 (dashed line in the main panel and in the inset). Surprisingly, the obtained total reflectivity $R_{\text {tot }}$ corresponds very well to the one of the static case. For the smaller velocities $(v=0.2 \mathrm{~m} / \mathrm{s}, v=2 \mathrm{~m} / \mathrm{s})$, beyond first order sidebands are negligible. For $v=6 \mathrm{~m} / \mathrm{s}$, also peaks of higher order become relevant. Here the reflectivity is distributed over many more sidebands.

Our calculations show that a particle, being reflected on an oscillating surface, may gain energy or transfer energy to the surface, if the oscillating energy $\hbar \omega$ is of the order of the energy of the particle. In a possible experimental investigation of this effect, the different scattering channels could be addressed independently and used to improve the precision of the measurement. Even though oscillating membranes are commercially available [20], quantum reflection of matter waves from an oscillating surface has not yet been experimentally investigated to the best of our knowledge. 


\section{Conclusion}

In this paper we studied the quantum reflectivity of atoms from a periodically oscillating surface for the case of a realistic setup. Our numerical method integrates the timedependent Schrödinger equation and controls very well the absorbed part of the incoming wave packet. This allows us to precisely estimate the quantum reflectivity by an average over the regularization parameter $x_{0}$ of the potential. In the computed momentum distribution of the reflected particle sidebands arise, which correspond to an energy transfer of multiples of the oscillator energy $\hbar \omega$. The relative contribution of the different sidebands depends strongly on the incoming velocity of the particle. For large velocities, the first order loss channel $R_{-1}$ becomes the dominant contribution. This may be exploited in future experiments to slow down atomic or molecular beams. For example, the experiment described in [9] is ideally suited to study quantum reflection from an oscillating membrane as proposed here. Although our calculation so far deals with a one-dimensional setup, we are confident that we can generalize our method to a two-dimensional geometry.

\section{Acknowledgments}

We thank with great pleasure the Heidelberg Center for Quantum Dynamics for funding this project. Furthermore, we are grateful for support by the DFG (FOR760), the Helmholtz Alliance Program EMMI (HA-216), and the HGSFP (GSC 129/1).

\section{References}

[1] Izrailev F M 1990 Physics Reports 196299 - 392

[2] Breuer H P and Holthaus M 1991 Annals of Physics $211249-291$

[3] Koch P and van Leeuwen K 1995 Physics Reports 255289 - 403

[4] Buchleitner A, Delande D and Zakrzewski J 2002 Physics Reports 368409 - 547

[5] Blanes S, Casas F, Oteo J and Ros J 2009 Physics Reports 470151 - 238

[6] Krug A and Buchleitner A 2001 Phys. Rev. Lett. 86(16) 3538-3541

[7] Kolovsky A R and Buchleitner A 2003 Phys. Rev. E 68(5) 056213

[8] Shimizu F 2001 Phys. Rev. Lett. 86 987-990

[9] Druzhinina V and DeKieviet M 2003 Phys. Rev. Lett. 91(19) 193202

[10] Zhao B S, Schewe H C, Meijer G and Schöllkopf W 2010 Phys. Rev. Lett. 105(13) 133203

[11] Zhao B S, Meijer G and Schöllkopf W 2011 Science 331 892-894

[12] DeKieviet M, Jentschura U D and Lach G 2011 Modern Experiments on Atom-Surface Casimir Physics Casimir Physics (Lecture Notes in Physics vol 834) ed Dalvit D, Milonni P, Roberts D and da Rosa F (Springer Berlin Heidelberg) pp 393-418

[13] Wimberger S 2011 The European Physical Journal D 63 1-2

[14] Pasquini T A, Saba M, Jo G B, Shin Y, Ketterle W, Pritchard D E, Savas T A and Mulders N 2006 Phys. Rev. Lett. 97(9) 093201

[15] Gierling M, Schneeweiss P, Visanescu G, Federsel P, Häffner M, Kern D, Judd T E, Günther A and Fortágh J 2011 Nature Nanotech. 6 446-451

[16] Fink M, Eiglsperger J, Friedrich H and Madroñero J 2011 The European Physical Journal D 63 $33-39$ 
[17] Kalman O, Kiss T, Fortágh J and Domokos P 2012 Nano Lett. 12 435-439

[18] Fink M, Müller T, Eiglsperger J and Madroñero J 2013 Euro. Phys. Lett. in press

[19] Byrd T A, Ivory M K, Pyle A J, Aubin S, Mitchell K A, Delos J B and Das K K 2012 Phys. Rev. A 86(1) 013622

[20] Zwickl B M, Shanks W E, Jayich A M, Yang C, Bleszynski Jayich A C, Thompson J D and Harris J G E 2008 Applied Physics Letters 92103125

[21] Friedrich H, Jacoby G and Meister C G 2002 Phys. Rev. A 65(3) 032902

[22] Henneberger W C 1968 Phys. Rev. Lett. 21(12) 838-841

[23] Press W H, Flannery B P, Teukolsky S A and Vetterling W T 1988 Numerical recipes repr. ed (Cambridge: Cambridge Univ. Pr.)

[24] Wimberger S, Mannella R, Morsch O and Arimondo E 2005 Phys. Rev. Lett. 94(13) 130404

[25] Jones E, Oliphant T, Peterson P et al. 2001-2011 SciPy: Open source scientific tools for Python URL http://www.scipy.org/

[26] Jeske F, Stöferle T and DeKieviet M 2011 The European Physical Journal D 63 25-32 